Hardy-Ramanujan Journal

Vol. 33 (2010) pp. 23-31

\title{
AN ESTIMATE FOR THE MELLIN TRANSFORM OF HARDY'S FUNCTION
}

\author{
Matti JutilA
}

\section{Introduction}

Let

$$
Z(t)=\zeta\left(\frac{1}{2}+i t\right) \chi\left(\frac{1}{2}+i t\right)^{-1 / 2}
$$

be Hardy's function with $\chi(s)=2^{s} \pi^{s-1} \sin \left(\frac{1}{2} s \pi\right) \Gamma(1-s)$ as in the functional equation $\zeta(s)=\chi(s) \zeta(1-s)$ for Riemann's zeta-function, and define the modified Mellin transform

$$
\mathcal{M}(s)=\int_{1}^{\infty} Z(x) x^{-s} \mathrm{~d} x, \quad \sigma>1 .
$$

This can be analytically continued to an entire function, as shown in [5] as a consequence of the fact that $\left(2^{1-w}-1\right) \zeta(w)$ is an entire function. In fact, $\mathcal{M}(s)$ is related to this function with $w=2 s-\frac{1}{2}$. Thus, it is to be expected that $|\mathcal{M}(s)|$ should be comparable with $\left|\zeta\left(2 s-\frac{1}{2}\right)\right|$ in some $s$-domain, in particular $\mathcal{M}\left(\frac{1}{2}+i t\right) \ll(|t|+1)^{1 / 6+\varepsilon}$. Indeed, this is a special case of the following theorem which is a sharpening of Theorem 4 of [3].

Theorem. Let $\sigma_{1}$ and $\sigma_{2}$ be fixed numbers such that $\frac{1}{4}<\sigma_{1}<\sigma_{2}$. Then for $\sigma_{1} \leq \sigma \leq \sigma_{2}$ and any fixed $\varepsilon>0$ we have

$$
\mathcal{M}(\sigma+i t) \ll \tau^{5 / 6-(4 / 3) \sigma+\varepsilon}+\tau^{2 / 3-\sigma}+\tau^{-1},
$$

where $\tau=\max (|t|, 1)$.

\section{A decomposition of $\mathcal{M}(s)$}

Following the argument in [4], define

$$
f(s, w)=\zeta(w) \chi(w)^{-1 / 2}\left(-\left(w-\frac{1}{2}\right)^{2}\right)^{-s / 2}
$$

and note that for $s=\sigma+i t$ with $\sigma>1$ we have

$$
\mathcal{M}(s)=-i \int_{1 / 2+i}^{1 / 2+i \infty} f(s, w) \mathrm{d} w .
$$


We suppose for a moment that $\sigma>\frac{3}{2}$ and restrict $t$ to an interval $T<t<2 T$ with $T$ sufficiently large. Note that (1.1) holds for smaller values of $t$ since $\mathcal{M}(s)$ is entire. We move the integration to the contour $C_{1} \cup C_{2} \cup C_{3}$ with

$C_{1}=\left[\frac{1}{2}+i, \frac{1}{2}+T^{2} i\right], \quad C_{2}=\left[\frac{1}{2}+T^{2} i, 1+\delta+T^{2} i\right], \quad C_{3}=\left[1+\delta+T^{2} i, 1+\delta+\infty i\right]$,

where $\delta$ is a small positive constant. We may suppose that $\delta<\sigma_{1}-\frac{1}{4}$. The meaning of $\delta$ will be fixed in the sequel, whereas $\varepsilon$ will stand generally for small constants, not necessarily the same at each occurrence. Let $\mathcal{M}_{i}(s)$ be the integral over $C_{i}$. The functions $\mathcal{M}_{1}(s)$ and $\mathcal{M}_{2}(s)$ are entire by definition, and therefore $\mathcal{M}_{3}(s)$ is entire as well since $\mathcal{M}(s)$ is entire. We need estimates for the $\mathcal{M}_{i}(s)$ in the domain

$$
D=\left\{\sigma+i t \mid \sigma_{1}<\sigma<\sigma_{2}, T<t<2 T\right\}
$$

The condition $\sigma>\frac{3}{2}$ was imposed to guarantee the convergence of the integral $\mathcal{M}_{3}(s)$. However, we are going to decompose $\mathcal{M}_{3}(s)$ into parts which are holomorphic in $D$, and therefore the definition of $\mathcal{M}_{3}(s)$ will make sense as such in $D$ without any analytic continuation.

To begin with, we simplify the integrand. Writing $w=u+i x$, we have

$$
f(s, w)=\zeta(u+i x) \chi(u+i x)^{-1 / 2} x^{-s}\left(1-\left(u-\frac{1}{2}\right) \frac{2 i}{x}-\left(u-\frac{1}{2}\right)^{2} \frac{1}{x^{2}}\right)^{-s / 2} .
$$

Recall the approximation (see [2], eq. (1.25))

$$
\chi(a+i x)=\chi_{0}(a+i x)\left(1+O\left(x^{-1}\right)\right)
$$

with

$$
\chi_{0}(a+i x)=(2 \pi / x)^{a+i x-1 / 2} e^{i(x+\pi / 4)},
$$

where $a$ is bounded and $x \geq x_{0}>0$. Then, using a standard estimate for the zeta-function, we see that

$$
\mathcal{M}_{2}(s) \ll T^{1 / 2-2 \sigma+\delta} .
$$

Turning to the estimation of $\mathcal{M}_{1}(s)$ and $\mathcal{M}_{3}(s)$, we show next that $\chi(w)$ can be replaced by $\chi_{0}(w)$ in the respective integrand. In the case of $\mathcal{M}_{1}(s)$, note that for $s \in D$ we have

$$
\int_{1}^{T^{2}} \zeta\left(\frac{1}{2}+i x\right)\left(\chi\left(\frac{1}{2}+i x\right)^{-1 / 2}-\chi_{0}\left(\frac{1}{2}+i x\right)^{-1 / 2}\right) x^{-s} \mathrm{~d} x \ll T^{1-2 \sigma} \log ^{2} T+T^{-1},
$$

which is an admissible error term since $1-2 \sigma<\frac{5}{6}-\frac{4}{3} \sigma$ for $\sigma>\frac{1}{4}$. To verify this, integrate by parts with respect to the factor $x^{-s}$, applying (2.2) together its differentiated version

$$
\chi^{\prime}(a+i x)=\chi_{0}^{\prime}(a+i x)\left(1+O\left(x^{-1}\right)\right)
$$


which follows from (2.2) and (2.3) by Cauchy's integral formula. Also, a straightforward estimation shows that $\chi$ can be replaced by $\chi_{0}$ in $\mathcal{M}_{3}(s)$ with an error $\ll T^{1 / 2+\delta-2 \sigma}$.

We simplify the integrand of $\mathcal{M}_{3}(s)$ still a bit. The factor $(\cdots)^{-s / 2}$ in $(2.1)$ is

$$
1+i s x^{-1}\left(u-\frac{1}{2}\right)+O\left(T x^{-2}\right) .
$$

The contribution of the error term to $\mathcal{M}_{3}(s)$ is $\ll T^{-1 / 2-2 \sigma+\delta}$. The second term gives $s \tilde{\mathcal{M}}_{3}(s+1)$, up to a constant factor, where $\tilde{\mathcal{M}}_{3}(s)$ denotes a modification of $\mathcal{M}_{3}(s)$ with $f(s, w)=f(s, u+i x)$ replaced by

$$
f_{0}(s, w)=\zeta(w) \chi_{0}(w)^{-1 / 2} x^{-s} .
$$

We are going to see that the estimate (1.1) with the term $\tau^{-1}$ omitted holds for $\tilde{\mathcal{M}}_{3}(s)$, and therefore $s \tilde{\mathcal{M}}_{3}(s+1)$ is of the desired order. Hence, all in all, it remains to estimate $\tilde{\mathcal{M}}_{3}(s)$ instead of $\mathcal{M}_{3}(s)$, and for simplicity we denote $\tilde{\mathcal{M}}_{3}(s)$ again by $\mathcal{M}_{3}(s)$.

The zeta-factor $\zeta(w)=\zeta(u+i x)$ will be written in terms of summands $n^{-u-i x}$ (approximately in $\mathcal{M}_{1}(s)$ and precisely in $\mathcal{M}_{3}(s)$ ) and therefore $\mathcal{M}_{1}(s)$ and $\mathcal{M}_{3}(s)$ amount to integrals of the function

$$
x^{-a} e^{i \varphi(x, n)}
$$

with $a=\sigma$ or $a=\sigma-\frac{1}{4}-\frac{1}{2} \delta$ and

$$
\varphi(x, n)=\frac{1}{2} x \log (x / 2 \pi)-x / 2-x \log n-t \log x .
$$

We keep $s$ fixed for a moment and ignore the $t$-dependence in the notation. These exponential integrals will be treated by standard methods: the saddle point method and the first or second derivative tests. The condition for the saddle point $x_{0}=$ $x_{0}(n)$ is $\varphi_{x}\left(x_{0}(n), n\right)=0$, that is

$$
\log \left(x_{0} / 2 \pi n^{2}\right)=2 t / x_{0} .
$$

Note that

$$
e^{i \varphi\left(2 \pi n^{2}, n\right)}=(-1)^{n}\left(2 \pi n^{2}\right)^{-i t} .
$$

The equation (2.6) has a unique positive solution $x_{0}$. The functions $x_{0}(n)$ and

$$
\psi(n)=\varphi\left(x_{0}(n), n\right),
$$

which occur in connection with the saddle point method, will be discussed in the next section, in addition to some results of general nature. 


\section{Lemmas}

There are various versions of the saddle point method in literature; the following (see [6], p. 71) suffices for our purposes.

Lemma 1. Suppose that the functions $f(x)$ and $g(x)$ defined on the interval $[a, b]$ satisfy the following conditions: $f(x)$ is real-valued; $f^{(4)}(x)$ and $g^{\prime \prime}(x)$ are continuous; there exist numbers $H, U$, and $A$ such that $H>0, A<U, 0<b-a \leq U$, and

$$
\begin{gathered}
A^{-1} \ll f^{\prime \prime}(x) \ll A^{-1}, \quad f^{(3)}(x) \ll A^{-1} U^{-1}, \quad f^{(4)}(x) \ll A^{-1} U^{-2}, \\
g(x) \ll H, \quad g^{\prime}(x) \ll H U^{-1}, \quad g^{\prime \prime}(x) \ll H U^{-2} .
\end{gathered}
$$

Suppose, moreover, that $f^{\prime}(c)=0$ for $c \in[a, b]$. Then

$$
\begin{gathered}
\int_{a}^{b} g(x) e^{2 \pi i f(x)} d x=e^{\pi i / 4} \frac{g(c) e^{2 \pi i f(c)}}{\sqrt{f^{\prime \prime}(c)}}+O\left(H A U^{-1}\right)+ \\
+O\left(H \min \left(\left|f^{\prime}(a)\right|^{-1}, \sqrt{A}\right)\right)+O\left(H \min \left(\left|f^{\prime}(b)\right|^{-1}, \sqrt{A}\right)\right) .
\end{gathered}
$$

If the saddle point $c$ does not exist but the other conditions are satisfied, then (3.3) holds with the first two terms on the right omitted.

In [6], this lemma was formulated under the assumption that a saddle point really exists. Otherwise, one may use simply the first or second derivative test (say in the form of Lemmas 5.1.2 and 5.1.3 in [1]) to verify the last mentioned assertion.

Lemma 2. Let $x_{0}=x_{0}(n)$ and $\psi(n)$ be defined by (2.6) and (2.8). Then

$$
\begin{gathered}
x_{0}(n) \geq 2 \pi n^{2}, \\
\max \left(n^{2}, t / \log t\right) \ll x_{0}(n) \ll t \text { for } n \ll \sqrt{t},
\end{gathered}
$$

and

$$
x_{0}(n)=2 \pi n^{2}+2 t P\left(\frac{t}{n^{2}}\right) \text { for } n \gg \sqrt{t}
$$

where $P(x)=1+a_{1} x+\ldots$ is a power series converging in a certain interval $|x|<r$, for some positive constant $r$. Also,

$$
\begin{gathered}
x_{0}^{\prime}(n)=\left(1+\frac{2 t}{x_{0}}\right)^{-1} \frac{2 x_{0}}{n}, \\
\psi^{\prime}(n)=-\frac{x_{0}(n)}{n}, \\
\psi^{\prime \prime \prime}(n)=-\left(\frac{4 x_{0}^{3}+16 t^{2} x_{0}}{\left(x_{0}+2 t\right)^{3}}\right) \frac{t}{n^{3}} .
\end{gathered}
$$


If $n \gg \sqrt{t}$, then

$$
\begin{gathered}
\frac{d}{d n}\left(\psi(n)+\pi n^{2}\right)=-\frac{2 t}{n} P\left(\frac{t}{n^{2}}\right) \asymp \frac{t}{n}, \\
\frac{d^{2}}{d n^{2}}\left(\psi(n)+\pi n^{2}\right)=\frac{2 t}{n^{2}} Q\left(\frac{t}{n^{2}}\right) \asymp \frac{t}{n^{2}},
\end{gathered}
$$

where $Q(x)$ with $Q(0) \neq 0$ is a power series converging for $|x|<r$.

Proof. The inequality (3.4) follows immediately from (2.6). Also, (3.5) follows easily from (2.6) if we write it as

$$
\log \left(t / 2 \pi n^{2}\right)=2 t / x_{0}+\log \left(t / x_{0}\right) .
$$

For a proof of (3.6), consider the function $y=y(x)$ defined implicitly by the equation

$$
y e^{x y}-1=0
$$

with $y(0)=1$. By the implicit function theorem, $y$ can be solved uniquely in a certain interval $|x|<r$, where it can be written as a power series $y(x)=P_{0}(x)=$ $1-x+\frac{3}{2} x^{2}+\cdots$. Now, writing (2.6) in the form (3.12) with $y=2 \pi n^{2} / x_{0}, x=t / \pi n^{2}$, we see that

$$
2 \pi n^{2} / x_{0}=P_{0}\left(t / \pi n^{2}\right)
$$

if $t / n^{2}$ is sufficiently small. Hence, taking inverses, we have

$$
\frac{x_{0}}{2 \pi n^{2}}=1+\frac{t}{\pi n^{2}}-\frac{1}{2}\left(\frac{t}{\pi n^{2}}\right)^{2}+\cdots,
$$

and (3.6) follows.

An implicit differentiation of the formula (2.6) gives (3.7). Likewise, differentiation of (2.8) yields (3.8) in view of (2.5) and the definition of the saddle point $x_{0}(n)$. Now (3.9) can be verified on differentiating (3.8) twice and using (3.7) to eliminate the derivatives $x_{0}^{\prime}(n)$. Finally, to verify (3.10), combine (3.6) and (3.8), and (3.11) then follows by differentiation.

Lemma 3. Let $0<\sigma_{0} \leq \sigma \ll 1$ and $t<2 \pi x / C$, where $C>1$ is a constant. Then

$$
\zeta(s)=\sum_{n \leq x} n^{-s}-x^{1-s} /(1-s)+O\left(x^{-\sigma}\right) .
$$

Moreover, if $|t|<\pi x / C$, then

$$
\left(1-2^{1-s}\right) \zeta(s)=\sum_{n \leq x}(-1)^{n-1} n^{-s}+O\left(x^{-\sigma}\right) .
$$

Proof. The formula (3.13) is well-known (see [7], Theorem 4.11), and (3.14) is an easy corollary: write

$$
\sum_{n \leq x}(-1)^{n-1} n^{-s}=\sum_{n \leq x} n^{-s}-2^{1-s} \sum_{n \leq x / 2} n^{-s}
$$

and apply (3.13) to the sums on the right.

The following well-known lemma (see [7], Theorem 5.11) might be called a "third derivative test" for exponential sums. 
Lemma 4. Let $f(x)$ be real and have continuous derivatives up to the third order, and let $\lambda_{3} \leq f^{\prime \prime \prime}(x) \leq h \lambda_{3}$, or $\lambda_{3} \leq-f^{\prime \prime \prime}(x) \leq h \lambda_{3}$ and $b-a \geq 1$. Then

$$
\sum_{a<n \leq b} e^{2 \pi i f(n)}=O\left(h^{1 / 2}(b-a) \lambda_{3}^{1 / 6}\right)+O\left((b-a)^{1 / 2} \lambda_{3}^{-1 / 6}\right) .
$$

\section{Estimation of $\mathcal{M}_{1}(s)$}

In Sec. 2, the estimation of $\mathcal{M}_{1}(s)$ was reduced to

$$
\int_{1}^{T^{2}} \zeta\left(\frac{1}{2}+i x\right) \chi_{0}\left(\frac{1}{2}+i x\right)^{-1 / 2} x^{-s} \mathrm{~d} x
$$

Repeated integration by parts with respect to the factor $x^{-s}$ shows that the integral over $\left[1, T_{0}\right]$ with $T_{0}=T^{1-\varepsilon}$ is $\ll T^{-1}+T^{1 / 6-\sigma+\varepsilon}$. Therefore it suffices to estimate the integral

$$
\int_{T_{0}}^{T^{2}} \zeta\left(\frac{1}{2}+i x\right) \chi_{0}\left(\frac{1}{2}+i x\right)^{-1 / 2} x^{-s} \mathrm{~d} x
$$

We write here

$$
\zeta\left(\frac{1}{2}+i x\right)=\sum_{n \leq x} n^{-1 / 2-i x}+O\left(x^{-1 / 2}\right)
$$

using (3.13); note that the second term in that formula could be absorbed into the error term in the present case. The contribution of the error term to the integral (4.1) is $\ll T^{1-2 \sigma+\varepsilon}+T^{1 / 2-\sigma+\varepsilon}$ which is negligible.

The main part of our integral comes from the zeta-sum on the right of (4.2). We have to estimate the sum

$$
\sum_{1 \leq n<T^{2}} n^{-1 / 2} \int_{\max \left(T_{0}, n\right)}^{T^{2}} x^{-\sigma} e^{i \varphi(x, n)} \mathrm{d} x
$$

Neighborhoods of the saddle points $x_{0}(n)$ lying in the range of integration produce the most significant contribution to (4.3). Moreover, if $x_{0}(n)$ approaches $T^{2}$, then the corresponding error term in Lemma 1 increases, and hence this case must be treated with some care. Therefore we consider separately the following cases:

(i) $x_{0}(n) \leq \frac{2}{3} T^{2}, \frac{1}{2} x_{0}(n) \leq x \leq \frac{3}{2} x_{0}(n)$,

(ii) $\frac{2}{3} T^{2}<x_{0}(n)<2 T^{2}, \frac{1}{2} T^{2} \leq x \leq T^{2}$,

(iii) the remaining pairs $(n, x)$ with $1 \leq n<T^{2}$ and $\max \left(T_{0}, n\right) \leq x \leq T^{2}$.

In the cases (i) and (ii), we apply Lemma 1 with $x$ running over the given interval and with

$$
\begin{gathered}
g(x)=x^{-\sigma}, \quad f(x)=\varphi(x, n) / 2 \pi \\
A=x_{0}(n)\left(1+T / x_{0}(n)\right)^{-1}, \quad U=x_{0}(n), \quad H=x_{0}(n)^{-\sigma}, \quad c=x_{0}(n) .
\end{gathered}
$$


Consider first the error terms of Lemma 1. In the case (i), we have $\left|\varphi_{x}\left(\frac{1}{2} x_{0}(n), n\right)\right| \gg$ 1 and $\left|\varphi_{x}\left(\frac{3}{2} x_{0}(n), n\right)\right| \gg 1$, so the error terms are $\ll x_{0}(n)^{-\sigma}$ and their contribution is

$$
\ll(T / \log T)^{-\sigma} \sum_{1 \leq n \ll T^{1 / 2}} n^{-1 / 2}+\sum_{T^{1 / 2} \ll n \ll T} n^{-1 / 2-2 \sigma} \ll T^{1 / 4-\sigma+\varepsilon}
$$

by Lemma 2.

In the case (ii), we use the estimate

$$
\left|\varphi_{x}\left(T^{2}, n\right)\right| \gg\left|x_{0}(n)-T^{2}\right| T^{-2}
$$

which follows from the definition of the saddle point together with the estimate

$$
\varphi_{x x}(x, n) \asymp x^{-1} \asymp T^{-2} \text { for } x \asymp T^{2}, n \asymp T \text {. }
$$

Noting also that $x_{0}^{\prime}(n) \asymp T$ by $(3.7)$, we see that the contribution of the error terms in question can be estimated as above.

In the case (iii), we have $\left|\varphi_{x}(x, n)\right| \gg 1$, so by the first derivative test the corresponding contribution is

$$
T_{0}^{-\sigma} \sum_{1 \leq n \leq T_{0}} n^{-1 / 2}+\sum_{T_{0} \leq n<T^{2}} n^{-1 / 2-\sigma} \ll T^{1 / 2-\sigma+\varepsilon}+T^{1-2 \sigma+\varepsilon} .
$$

Consider finally the saddle-point terms, which occur when $x_{0}(n) \leq T^{2}$. Then $n \ll T$ by Lemma 2 , and the contribution of these terms is

$$
\sqrt{2 \pi} e^{\pi i / 4} \sum_{1 \leq n \ll T} n^{-1 / 2} x_{0}(n)^{1-\sigma} e^{i \psi(n)}\left(\frac{1}{2} x_{0}(n)+t\right)^{-1 / 2} .
$$

We decompose the sum into subsums over intervals $\left[N, N^{\prime}\right]$ with $N<N^{\prime} \leq 2 N$ and $N \ll T$, and it suffices to estimate these subsums individually.

First, if $N \ll T^{1 / 3}$, we have $x_{0}(n) \asymp T / \log T$, and by a trivial estimation we see that the corresponding sum is

$$
\ll N^{1 / 2} T^{1 / 2-\sigma+\varepsilon} \ll T^{2 / 3-\sigma+\varepsilon} .
$$

Next, if $T^{1 / 3} \ll N \ll T^{2 / 3}$, we use Lemma 4 . Since $x_{0}(n)$ is a smooth function of $n$ by (3.7), we have essentially exponential sums $\sum e^{i \psi(n)}$ to deal with. By (3.9) and the bounds for $x_{0}(n)$ obtained in Lemma 2, we have $\left|\psi^{\prime \prime \prime}(n)\right| \asymp \lambda_{3}$ with

$$
T /\left(N^{3} \log T\right) \ll \lambda_{3} \ll T / N^{3} .
$$

Let $x_{0}(n) \asymp X$ for $N \leq n \leq N^{\prime}$; then $T / \log T \ll X \ll T$ for $T^{1 / 3} \ll N \ll T^{1 / 2}$ and $X \asymp N^{2}$ otherwise. Using summation by parts and Lemma 4 , we see that our sum is

$$
\begin{gathered}
\ll N^{-1 / 2} X^{1 / 2-\sigma} T^{\varepsilon}\left(N \lambda_{3}^{1 / 6}+N^{1 / 2} \lambda_{3}^{-1 / 6}\right) \ll X^{1 / 2-\sigma} T^{1 / 6+\varepsilon} \\
\ll T^{5 / 6-(4 / 3) \sigma+\varepsilon}+T^{2 / 3-\sigma+\varepsilon} .
\end{gathered}
$$


Finally, if $T^{2 / 3} \ll N \ll T$, then $X \asymp N^{2}$. We write

$$
e^{i \psi(n)}=(-1)^{n} e^{i\left(\psi(n)+\pi n^{2}\right)}
$$

with an application of (3.10) and (3.11) in mind. Let us consider even and odd values of $n$ separately and use the exponent pair $\left(\frac{1}{2}, \frac{1}{2}\right)$ (see [2], p. 77). In this way, we see that

$$
\sum_{n \asymp N} e^{i \psi(n)} \ll T^{1 / 2}
$$

Hence our sum is

$$
\ll N^{1 / 2-2 \sigma} T^{1 / 2} \ll T^{5 / 6-(4 / 3) \sigma} .
$$

All in all, the contribution of the saddle point terms is bounded by the right hand side of (1.1). And as we saw above, the same is true for the error terms as well.

\section{Estimation of $\mathcal{M}_{3}(s)$}

As we showed in sec. 2 , the estimation of $\mathcal{M}_{3}(s)$ can be reduced to

$$
\int_{T^{2}}^{\infty} \zeta(1+\delta+i x) \chi_{0}(1+\delta+i x)^{-1 / 2} x^{-s} \mathrm{~d} x
$$

that is to

$$
\sum_{n=1}^{\infty} n^{-1-\delta} \int_{T^{2}}^{\infty} x^{1 / 4+\delta / 2-\sigma} e^{i \varphi(x, n)} \mathrm{d} x .
$$

This is similar to (4.3), and we argue much as in the preceding section.

Let us subdivide the relevant pairs $(n, x)$ into four classes by the following conditions:

(i) $\left|2 \pi n^{2}-T^{2}\right| \leq c T, \quad T^{2} \leq x \leq 2 T^{2}$,

(ii) $c T<\left|2 \pi n^{2}-T^{2}\right|<\frac{1}{2} T^{2}, \quad T^{2} \leq x \leq 2 T^{2}$,

(iii) $2 \pi n^{2} \geq \frac{3}{2} T^{2}, \quad \frac{2}{3}\left(2 \pi n^{2}\right) \leq x \leq \frac{3}{2}\left(2 \pi n^{2}\right)$,

(iv) the remaining pairs $(n, x)$ with $n \geq 1$ and $x \geq T^{2}$.

Here $c$ is a positive constant such that $x_{0}(n) \geq T^{2}+T$ for

$$
2 \pi n^{2}>T^{2}+c T
$$

and $x_{0}(n) \leq T^{2}-T$ for $2 \pi n^{2}<T^{2}-c T$; such a constant exists by (3.6). Thus the subdivision is motivated by the location of the saddle point $x_{0}(n)$ depending on $t$, but we preferred formulating the conditions in terms of $2 \pi n^{2}$ in order to avoid dependence on $t$.

The contribution of each class is holomorphic in $D$. This is clear in the cases (i) and (ii), and convergence considerations will show that the same is true for the cases (iii) and (iv) as well.

In the case (i), the number of relevant values of $n$ is finite, and their contribution to $(5.1)$ is $\ll T^{1 / 2-2 \sigma}$ by the second derivative test.

In the cases (ii) and (iii), we apply Lemma 1. The contribution of the saddle point terms to $(5.1)$ is

$$
\sqrt{2 \pi} e^{\pi i / 4} \sum_{n} n^{-1-\delta} x_{0}(n)^{5 / 4+\delta / 2-\sigma} e^{i \psi(n)}\left(\frac{1}{2} x_{0}(n)+t\right)^{-1 / 2},
$$


where the condition for $n$ is given by (5.2). Further, using (3.6), we have

$n^{-1-\delta} x_{0}(n)^{5 / 4+\delta / 2-\sigma}\left(\frac{1}{2} x_{0}(n)+t\right)^{-1 / 2}=\sqrt{2}(2 \pi)^{3 / 4+\delta / 2-\sigma} n^{1 / 2-2 \sigma}+O\left(T n^{-3 / 2-2 \sigma}\right)$.

The contribution of the error term to $(5.3)$ is $\ll T^{1 / 2-2 \sigma}$. Thus the estimation of (5.3) amounts to

$$
\sum_{n \gg T} n^{1 / 2-2 \sigma} e^{i \psi(n)}
$$

We write here

$$
e^{i \psi(n)}=e^{i \varphi\left(2 \pi n^{2}, n\right)} e^{i\left(\psi(n)-\varphi\left(2 \pi n^{2}, n\right)\right)} .
$$

By (2.7), this is further

$$
(-1)^{n}\left(2 \pi n^{2}\right)^{-i t} e^{i\left(\psi(n)+t \log \left(2 \pi n^{2}\right)+\pi n^{2}\right)} .
$$

Since

$$
\frac{d}{d n}\left(\psi(n)+t \log \left(2 \pi n^{2}\right)+\pi n^{2}\right) \ll t^{2} n^{-3}
$$

by (3.10), summation by parts reduces (5.4) to sums

$$
\sum_{T \ll n \ll N}(-1)^{n} n^{1 / 2-2 s} \ll T^{1 / 2-2 \sigma},
$$

the last step by (3.14).

Finally, the contribution of the pairs (iv) and the error terms of Lemma 1 in the cases (ii) and (iii) can be estimated by $\ll T^{1 / 2-2 \sigma+\delta}$. The calculations run as in the preceding section except that now our assumptions on $\sigma_{1}$ and $\delta$ are needed to ensure the convergence of our integrals in (iv).

The preceding estimations show that (1.1) holds for $\mathcal{M}_{3}(s)$, even with the last term $\tau^{-1}$ omitted. Now, combining the estimations in the last two sections with (2.4), we complete the proof of the theorem.

\section{REFERENCES}

[1] M. N. Huxley, Area, Lattice Points and Exponential Sums, Clarendon Press, Oxford, 1996.

[2] A. Ivić, The Riemann zeta-function, John Wiley and Sons, New York, 1985.

[3] A. Ivić, On the Mellin transforms of powers of Hardy's function, arXiv:1001.1824v2[math.NT], Hardy-Ramanujan J. 33 (2010) (to appear).

[4] M. Jutila, The Mellin transform of the square of Riemann's zeta-function, Periodica Math. Hung. 42 (2001), 179-190.

[5] M. Jutila, The Mellin transform of Hardy's funtion is entire, Mat. Zametki (to appear).

[6] A. A. Karatsuba and S. M. Voronin, The Riemann Zeta-Function, Walter de Gruyter, BerlinNew York, 1992.

[7] E. C. Titchmarsh, The theory of the Riemann zeta-function, 2nd ed., The Clarendon Press, Oxford, 1986.

Department of Mathematics, University of Turku, FI-20014 Turku, Finland; email: jutila@utu.fi 